\title{
Reliable teleportation in trapped ions
}

\author{
E. Solano ${ }^{12}$, C. L. Cesar ${ }^{1}$, R. L. de Matos Filho ${ }^{1}$, and N. Zagury ${ }^{1}$ \\ 1 Instituto de Física, Universidade Federal do Rio de Janeiro, Caixa Postal 68528, 21945-970 Rio de Janeiro, RJ, Brazil \\ 2 Sección Física, Departamento de Ciencias, Pontificia Universidad Católica del Perú, Apartado 1761, Lima, Peru
}

August 28, 2018

\begin{abstract}
We study a method for the implementation of a reliable teleportation protocol (theoretically, $100 \%$ of success) of internal states in trapped ions. The generation of the quantum channel (any of four Bell states) may be done respecting technical limitations on individual addressing and without claiming the Lamb-Dicke regime. An adequate Bell analyzer, that transforms unitarily the Bell basis into a completely disentangled one, is considered. Probable sources of error and fidelity estimations of the teleportation process are studied. Finally, we discuss experimental issues, proposing a scenario in which the present scheme could be implemented.
\end{abstract}

PACS. 03.67.Hk - 03.65.Bz - 42.50.Vk

\section{Introduction}

Entanglement, or non separability, is a natural consequence of linearity in quantum mechanics and its careful study is one of the relevant tasks in fundamentals of physics. Since Einstein-Podolski-Rosen seminal work [1], non local effects ' in two, or more, entangled particles have been the object of intense research. Local hidden variables theories, partially designed to avoid non intuitive explanations, led to results in contradiction with several experiments [2, 3]. As a consequence, the deterministic generation of entanglement in a pair of two-level particles, being this entangled bipartite system the simplest one, has become an important theoretical and experimental demand. In particular, there exists a fundamental interest in the generation of Bell states, a complete basis in a four dimensional Hilbert space made out of maximally entangled orthogonal states. Additionally, it has been shown that producing Bell states in two macroscopically distant particles is of extreme importance to experimentalists for the implementation of quantum teleportation [ [1, 5. 6, 6], quantum cryptography [7] and quantum computation [8,9].

Massive particles, like atoms or ions, are good for storing quantum information and photons are the natural messengers for communicating them. Controlled entanglement between massive particles has been achieved in the case of atoms crossing a high $Q$ cavity [10,11] and in the case of ions in a trap [12, 13].

Bell states provides the essential non local ingredient for performing quantum teleportation, where a disembodied transmission of an arbitrary state, from one system to another located at a remote place, is made through a classical and a quantum channel. After the original proposal of quantum teleportation 㮌 (hereafter called BBCJPW), many experimental efforts have been made in order to test teleportation of an arbitrary state of discrete [5] or continuous variables [6]. In all these experiments the particles involved were photons. The main difficulty for the implementation of the BBCJPW protocol for teleportation of discrete states is the complete measurement of a non-degenerate Bell operator [14]. This would require the projection of an input two-particle state on one of four maximally entangled orthogonal states and the production of a unique signal for each one. In fact, it has recently been shown that, in the case of teleportation of discrete variables, it is not possible to perform a complete Bell-operator measurement without an effective quantum interaction between the involved particles [15, 16]. Due to the lack of a photon-photon interaction, it seems impossible to obtain a reliable teleportation of photon polarization states that follows closely the original BBCJPW protocol. For this reason, it seems more promising to look for systems where effective quantum interactions between their components may be implemented more easily, like trapped ions or systems composed of atoms interacting with electromagnetic cavities [17, 18]. Teleportation with trapped ions has an additional important advantage: we are dealing with a single long living quantum system, confined into a very small region of space and that remains at our disposal for further manipulation. In this paper we propose a method for implementing a reliable teleportation protocol (theoretically, $100 \%$ of success) of an arbitrary internal state in trapped ions.

In Section II, we discuss a proposal for reliable teleportation of arbitrary two-level electronic states between ions kept in two well separated traps. This is accomplished by means of a Bell state as the quantum channel and the implementation of an adequate Bell analyzer using well 
determined unitary operations and projections, avoiding undesired entanglement of the internal states with the motional degrees of freedom. Due to the use of ion-ion quantum interaction, all the measurements needed in this scheme are performed exclusively on single ions. In this way, our Bell analyzer distinguishes efficiently the four Bell states. Numerical estimations of the fidelity of the teleportation process are also presented. In Section III, we consider some generalization of our scheme for implementing entanglement teleportation and entanglement swapping. In Section IV, we discuss relevant experimental issues for the practical realization of our teleportation scheme. Finally, we summarize our main results in Section V.

\section{Teleportation}

\subsection{Scheme}

Our procedure, along the lines of the BBCJPW protocol [《], can be sketched as follows (see Fig. 1): Trap A (Alice station) contains ion 1 with an arbitrary electronic state, $\alpha\left|\downarrow_{1}\right\rangle+\beta\left|\uparrow_{1}\right\rangle$ and trap $B$ (Bob station) contains ions 2 and 3 in a maximally entangled state, e.g. any of the four Bell states. Ion 2 is then transferred adiabatically to Alice station, who performs a suitable operation on the joint Hilbert space of ions 1 and 2 transforming unitarily the Bell base into a disentangled base. Subsequently, Alice measures their individual electronic states, completing the so called Bell analyzer. Then, Alice informs to Bob the result of her measurements, consisting in two bits of classical information. Bob uses them to perform one, out of four, specific unitary transformation on ion 3, whose electronic state is left at the original state of ion 1.

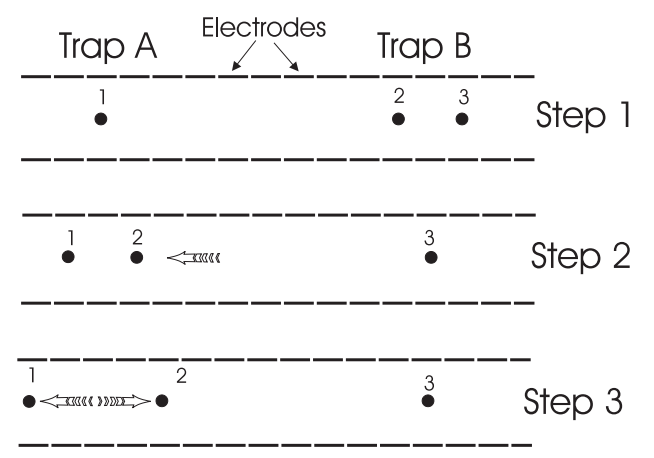

\subsection{Quantum channel}

The first task in the implementation of our teleportation scheme is the preparation of the quantum channel, i.e., the deterministic generation of an electronic Bell state in trap B. In a real situation we will deal with initial motional thermal states, where contributions of different vibrational Fock states, associated with the center of mass mode of frequency $\nu$ and with the stretch mode of frequency $\nu_{r}$, are relevant. Therefore, we will consider the general proposal of Ref. [19] for the generation of the four Bell states. According to it, one of the Bell states may be generated, with present techniques and not claiming the Lamb-Dicke regime, through the use of two pairs of Raman lasers acting on both ions. The two Raman laser pairs induce dispersive interactions between two long-living hyperfine levels, $|\downarrow\rangle$ and $|\uparrow\rangle$ (energy difference equal to $\hbar \omega_{0}$ ). One pair produces a dispersive transition to the $k^{\text {th }}$ blue sideband $\left(\omega_{I}=\omega_{o}+k \nu-\delta\right)$ while the other causes a dispersive transition to the $k^{t h}$ red sideband $\left(\omega_{I I}=\omega_{o}-k \nu+\delta\right)$. See Fig. 2 for a sketch of the energy level diagram.

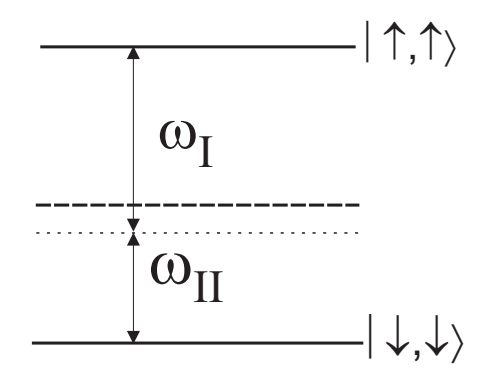

Fig. 2. Energy level diagram. A stimulated electronic transition is induced by means of two dispersive laser excitations with frequencies $\omega_{I}=\omega_{o}+k \nu-\delta$ and $\omega_{I I}=\omega_{o}-k \nu+\delta$ in such a way that $\omega_{I}+\omega_{I I}=2 \omega_{o}$.

This process is described by the general effective Hamiltonian 19

$$
\begin{aligned}
\widehat{H}_{\mathrm{eff}} & =\hbar \Omega_{k}\left[\widehat{S}_{+j} \widehat{S}_{+m} e^{2 i \phi}+(-1)^{k}\left(\widehat{S}_{+j} \widehat{S}_{-m} e^{i \phi_{o}}+\frac{1}{2}\right)\right] \\
& \times\left[\widehat{F}_{k}^{2}\left(\hat{n}-k, \hat{n}_{r}\right) \frac{\hat{n} !}{(\hat{n}-k) !}-\widehat{F}_{k}^{2}\left(\hat{n}, \hat{n}_{r}\right) \frac{(\hat{n}+k) !}{\hat{n} !}\right] \\
& + \text { H.c. }
\end{aligned}
$$

where $\Omega_{k}=2|\Omega|^{2}(i \eta)^{2 k} / \delta$. Here, $\Omega$ is the Rabi frequency associated with each Raman pair and $\eta$ is the CM LambDicke parameter. $\widehat{S}_{+m}=\left|\uparrow_{m}\right\rangle\left\langle\downarrow_{m}\right|$ and $\widehat{S}_{-m}=S_{+m}^{\dagger}$ are the electronic raising and lowering operators acting on ion $m, \phi$ is the effective phase, taking as equal, of each Raman laser pair and $\phi_{0}$ is the known phase difference associated with the equilibrium separation of the two ions. $\hat{n}$ and $\hat{n_{r}}$ are the harmonic oscillator number operators associated
Fig. 1. Sketch of the trap configuration for teleportation of the electronic state of ion 1 to ion 3 . In the first step, ion 1 has an arbitrary electronic state, while ions 2 and 3 are in an Bell state. In step 2, the second ion is transported to trap $A$, where it undergoes entanglement with ion 1. As a last step, ions 1 and 2 are separated so that their individual states can be measured via fluorescence. 
with the center of mass $(\mathrm{CM})$ and the relative modes, respectively. Also,

$$
\widehat{F}_{k}\left(\hat{n}, \hat{n}_{r}\right)=\sum f_{k}\left(n, n_{r}\right)\left|n, n_{r}\right\rangle\left\langle n, n_{r}\right|
$$

with

$$
f_{k}\left(n, n_{r}\right)=e^{-\left(\eta^{2}+\eta_{r}^{2}\right) / 2} \frac{n !}{(n+k) !} L_{n}^{k}\left(\eta^{2}\right) L_{n_{r}}^{0}\left(\eta_{r}^{2}\right),
$$

where

$$
L_{m}^{k}(x)=\sum_{\ell=0}^{m}(-1)^{\ell}\left(\begin{array}{c}
m+k \\
m-\ell
\end{array}\right) \frac{x^{\ell}}{\ell !}
$$

are associated Laguerre polynomials. The first term of Eq. (11) and its Hermitian conjugate, acting only in the subspace determined by the states $\{|\downarrow \downarrow\rangle,|\uparrow \uparrow\rangle\}$, describe two-photon processes leading to the simultaneous excitation or deexcitation of the electronic states of the two ions. The second term and its Hermitian conjugate, acting exclusively in the subspace spanned by the states $\{|\downarrow \uparrow\rangle,|\uparrow \downarrow\rangle\}$, describe processes where one ion undergoes a transition from the ground to the excited electronic state and the other ion makes a transition in the inverse direction, both processes taking place simultaneously. The third term is a self energy term where the energy shifts in the two effective ionic levels are equal and in the same direction, just producing a time-dependent overall phase in the states evolution, as we will see below. The vibrational part of this Hamiltonian, completely factorized on the right, contains only number operators that will not change the population of a two mode Fock state basis $\left|n, n_{r}\right\rangle$. The first (second) term of the vibrational part has its origin in a virtual two step process: in the first one, $k$ vibrational quanta are annihilated (created) and in the second one, the same number of vibrational quanta are created (annihilated), preserving the number of motional quanta. It is important to note that, when the vibrational part of the initial state is the ground state $(n=0)$, the first term of the vibrational part vanishes due to the fact that, in this case, is impossible to annihilate a motional quantum. We may also notice that there is no effective coupling when $k=0$, that is, when no collective mode is excited together with the intermediate virtual electronic levels.

In particular, we are interested in the temporal evolution of initial states of the form $|\downarrow, \downarrow\rangle_{n, n_{r}} \equiv|\downarrow, \downarrow\rangle \otimes$ $\left|n, n_{r}\right\rangle,|\uparrow, \uparrow\rangle_{n, n_{r}},|\downarrow, \uparrow\rangle_{n, n_{r}}$ and $|\uparrow, \downarrow\rangle_{n, n_{r}}$. So, for an interaction time $t$ we have

$$
\begin{aligned}
& e^{-\frac{i}{\hbar} \widehat{H}_{\mathrm{eff}} t}|\downarrow, \downarrow\rangle_{n, n_{r}}=e^{-i(-1)^{k} \Omega_{n n_{r}}^{k} t}\left[\cos \left(\left|\Omega_{n n_{r}}^{k}\right| t\right)|\downarrow, \downarrow\rangle\right. \\
& \left.+i(-1)^{k} e^{2 i \phi} \sin \left(\left|\Omega_{n n_{r}}^{k}\right| t\right)|\uparrow, \uparrow\rangle\right]_{n, n_{r}} \\
& e^{-\frac{i}{\hbar} \widehat{H}_{\text {eff }} t}|\uparrow, \uparrow\rangle_{n, n_{r}}=e^{-i(-1)^{k} \Omega_{n n_{r}}^{k} t}\left[\cos \left(\left|\Omega_{n n_{r}}^{k}\right| t\right)|\uparrow, \uparrow\rangle\right. \\
& \left.+i(-1)^{k} e^{-2 i \phi} \sin \left(\left|\Omega_{n n_{r}}^{k}\right| t\right)|\downarrow, \downarrow\rangle\right]_{n, n_{r}} \\
& e^{-\frac{i}{\hbar} \widehat{H}_{\text {eff }} t}|\downarrow, \uparrow\rangle_{n, n_{r}}=e^{-i(-1)^{k} \Omega_{n n_{r}}^{k} t}\left[\cos \left(\left|\Omega_{n n_{r}}^{k}\right| t\right)|\downarrow, \uparrow\rangle\right. \\
& \left.+i e^{i \phi_{o}} \sin \left(\left|\Omega_{n n_{r}}^{k}\right| t\right)|\uparrow, \downarrow\rangle\right]_{n, n_{r}} \\
& e^{-\frac{i}{\hbar} \widehat{H}_{\text {eff }} t}|\uparrow, \downarrow\rangle_{n, n_{r}}=e^{-i(-1)^{k} \Omega_{n n_{r}}^{k} t}\left[\cos \left(\left|\Omega_{n n_{r}}^{k}\right| t\right)|\uparrow, \downarrow\rangle\right. \\
& \left.+i e^{-i \phi_{o}} \sin \left(\left|\Omega_{n n_{r}}^{k}\right| t\right)|\downarrow, \uparrow\rangle\right]_{n, n_{r}},
\end{aligned}
$$

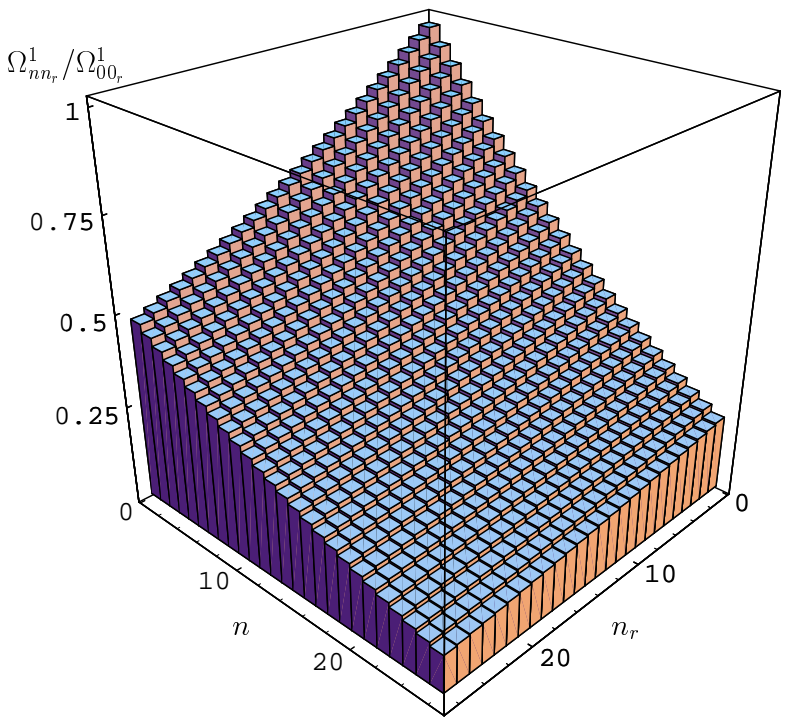

Fig. 3. Scaled Rabi frequency $\Omega_{n n_{r}}^{k}$ plotted as a function of $n$ and $n_{r}$ for $k=1$ and $\eta=0.15$. We stress the fact that we have a different positive Rabi frequency for each different pair $(0,0)<\left(n, n_{r}\right)<(25,25)$.

where

$$
\Omega_{n n_{r}}^{k}=\Omega_{k}\left[f_{k}^{2}\left(n-k, n_{r}\right) \frac{n !}{(n-k) !}-f_{k}^{2}\left(n, n_{r}\right) \frac{(n+k) !}{n !}\right]
$$

are effective Rabi frequencies. In Fig. 3, we plot their dependence, for the particular case $k=1$ and $\eta=0.15$, with the $\mathrm{CM}$ and relative motion quanta number, $\hat{n}$ and $\hat{n_{r}}$. This dependence is relevant when considering realistic situations for the teleportation protocol.

It is easy to show that in the simpler case $k=1$ and in the Lamb-Dicke limit the effective Hamiltonian given in Eq. (1) reduces to

$$
H=\hbar\left|\Omega_{1}\right|\left[S_{+j} S_{+m} e^{2 i \phi}-S_{+j} S_{-m} e^{i \phi_{0}}-\frac{1}{2}\right]+H . c .
$$

Notice that, in this limit, this interaction is identical to the one developped by A. Sørensen and K. Mølmer [20], who first noticed the relevance of its independence on the vibrational quantum state of the ions. In the analytical description of the teleportation scheme we will use this particular Hamiltonian. Nevertheless, it is clear that when considering realistic situations in our simulations, as an initial motional thermal state, we will have to use the full $n$ and $n_{r}$ dependence given by the more general Hamiltonian presented in Eq. (11). For the preparation of the Bell state in trap $B$, we consider that the two ions, 2 and 3 , were previously cooled down to the Lamb-Dicke regime and stay in their electronic ground states. By letting the two Raman laser pairs interact with the ions during a time 
$\tau=\pi /\left(4\left|\Omega_{1}\right|\right)$, we prepare the Bell electronic state

$$
\left|\Phi_{23}\right\rangle=\frac{1}{\sqrt{2}}\left(\left|\downarrow_{2}, \downarrow_{3}\right\rangle-i e^{2 i \phi_{B}}\left|\uparrow_{2}, \uparrow_{3}\right\rangle\right),
$$

where $\phi_{B}$ is the effective phase of both Raman lasers pairs in trap $B$. We then carefully transfer ion 2 from trap $B$ to trap $A$, where it interacts with ion 1 . Possible mechanisms to make this transfer will be discussed in Section 4 , with the only necessary, and feasible, condition that no entanglement should occur between the electronic and motional states. We assume that after the transfer process the mean quantum number $\bar{n}$ associated to the vibrational modes will be small, so that the Lamb-Dicke approximation, $\bar{n} \eta^{2} \ll 1$, remains valid in both traps. If the transfer process itself causes excessive heating, sympathetic cooling techniques may be used to reduce $\bar{n}$. The total electronic state of ions 1, 2 and 3 is then given by

$$
\left|\Psi_{0}(1,2,3)\right\rangle=\left(\alpha\left|\downarrow_{1}\right\rangle+\beta\left|\uparrow_{1}\right\rangle\right)\left|\Phi_{23}\right\rangle .
$$

Now we want to consider, in a more realistic way, the generation of the maximally entangled state. For this purpose, we estimate the fidelity in the generation of the Bell state $\rho=\left|\Phi^{(+)}\right\rangle\left\langle\Phi^{(+)}\right|$when both ions are initially in their electronic ground state and in a collective two mode motional thermal state. By making use of Eq. (5) together with Eq. (伍), the fidelity $F(\tau)=\operatorname{Tr}\left\{\rho \rho^{\prime}(\tau)\right\}$ of the resulting state $\rho^{\prime}(\tau)$, after an interaction time $\tau$, is found to be

$$
\mathrm{F}(\tau)=\frac{1}{2}+\frac{1}{2} \sum_{n n_{r}} P_{n n_{r}} \sin \left(2\left|\Omega_{n n_{r}}^{1}\right| \tau\right),
$$

where $P n n_{r}$ is the initial two mode vibrational thermal distribution. We consider an exact $\pi / 4$-pulse associated with the effective Rabi frequency $\Omega_{00_{r}}^{1}$ given in Eq. (6), so that $\tau=\pi /\left(4 \Omega_{00_{r}}^{1}\right)$. For the cases where the vibrational modes are initially in a thermal distribution with mean number occupation $\bar{n}=0.2,1,5$ and, considering the same associated temperature, $\bar{n}_{r}=0.047,0.43,2.69$, the fidelity given by Eq. (10) is $F=0.999,0.988,0.880$, respectively. This high fidelity in the generation of the quantum channel permit us to continue confidently with the teleportation protocol.

It is important to mention that in a recent work [21] Sørensen and Mølmer develop a careful study of the fidelity in the generation of a Bell state. Even if they use an alternative interaction regime, in the search of a faster operation, we find relevant to mention that our fidelity estimations are similar. For example, under the same realistic conditions metioned above $(\bar{n}=0.2$ and $\eta=0.2)$, their analytical expresions give $F=0.999$, while it goes down to $F=0.9$ when $\bar{n}=5$ and the same $\eta=0.2$. This alternative scheme was already used for generating a Bell state in the NIST group [13], providing a first fundamental step for the implementation of a teleportation protocol in trapped ions.

\subsection{Bell analyzer}

If we follow closely the recipe of the BBCJPW protocol [⿰亻弋 our second task would be to perform a complete measurement, of the von Neumann type, on the subsystem of particles 1 and 2 in the Bell operator basis, that will confidently provide two bits of classical information. We propose here a practical way of obtaining this classical information by suitably entangling the electronic state of particles 1 and 2 and then measuring the individual electronic state of each ion. This substitutes the non-trivial requirement of a direct Bell measurement by that of monitoring the individual ion fluorescence. For achieving this goal, we first apply, on ions 1 and 2, a pulse of duration $\tau=\pi /\left(4\left|\Omega_{1}\right|\right)$, using the same excitation scheme as used before in trap $B$, transforming the total electronic state $\left|\Psi_{0}(1,2,3)\right\rangle$ into

$$
\begin{aligned}
|\Psi(1,2,3)\rangle= & \\
\frac{1}{2}\left\{-i e^{2 i \phi_{A}}\left|\uparrow_{1} \uparrow_{2}\right\rangle\right. & \otimes\left[\alpha\left|\downarrow_{3}\right\rangle+e^{2 i\left(\phi_{B}-\phi_{A}\right)} \beta\left|\uparrow_{3}\right\rangle\right] \\
+\left|\downarrow_{1} \downarrow_{2}\right\rangle & \otimes\left[\alpha\left|\downarrow_{3}\right\rangle-e^{2 i\left(\phi_{B}-\phi_{A}\right)} \beta\left|\uparrow_{3}\right\rangle\right] \\
+\left|\uparrow_{1} \downarrow_{2}\right\rangle & \otimes\left[\beta\left|\downarrow_{3}\right\rangle+e^{i\left(2 \phi_{B}+\phi_{0}\right)} \alpha\left|\uparrow_{3}\right\rangle\right] \\
+i e^{-i \phi_{0}}\left|\downarrow_{1} \uparrow_{2}\right\rangle & \left.\otimes\left[\beta\left|\downarrow_{3}\right\rangle-e^{i\left(2 \phi_{B}+\phi_{0}\right)} \alpha\left|\uparrow_{3}\right\rangle\right]\right\},
\end{aligned}
$$

where $\phi_{A}$ is the effective phase of both Raman laser pairs in trap $A$ and $\phi_{0}$ is the phase due to the equilibrium separation of the ions 1 and 2 in trap $A$. If we set, for simplicity, $\phi_{A}=\phi_{B}=\pi-\phi_{0} / 2$, it is clear that the determination of the energy state of ions 1 and 2 projects ion 3 on one of the 4 states $\alpha\left|\downarrow_{3}\right\rangle+\beta\left|\uparrow_{3}\right\rangle, \alpha\left|\downarrow_{3}\right\rangle-\beta\left|\uparrow_{3}\right\rangle, \beta\left|\downarrow_{3}\right\rangle+\alpha\left|\uparrow_{3}\right\rangle$, and $\beta\left|\downarrow_{3}\right\rangle-\alpha\left|\uparrow_{3}\right\rangle$. These four states corresponds to the original state or a $\pi$ rotation of it around the $z$ axis, the $x$ axis or the $y$ axis, respectively. If now Alice measures the electronic energy of each ion in her trap and send the result (2 bits of classical information) to Bob, he will need or not to make an additional operation (the corresponding inverse rotation around the $z, x$ or $y$ axis) on ion 3 . This completes the teleportation of the original arbitrary state from particle 1 to particle 3 . We remark that this scheme provides reliable teleportation [15], where we have theoretically $100 \%$ of success in distinguishing the four Bell states in our analyzer. This is also the case for atomcavity systems, as in the proposal discussed in Ref. [17], but impossible for photons in the absence of an effective photon-photon interaction.

It is important to note that, initially, ions 1 and 2 were not correlated and that the applied pulse entangles these particles, communicating ion 1 with the quantum channel already established in ions 2 and 3 . This is not in contradiction with the fact that this pulse, formally, transforms unitarily the Bell basis into a completely disentangled one, as we may see in Eq. (11), for the proper work of our Bell analyzer.

It is known that the quantum channel and a Bell analyzer in a teleportation scheme may be implemented using a product of a C-NOT gate and a Hadamard rotation [22], 


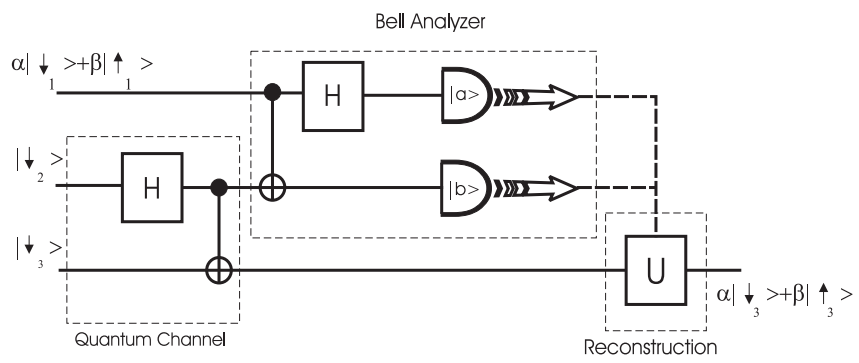

Fig. 4. Reliable teleportation quantum logic circuit associated with a typical teleportation scheme. The detectors may produce the following two-cbit information, $|a\rangle=\left\{\left|\downarrow_{1}\right\rangle,\left|\uparrow_{1}\right\rangle\right\}$ and $|b\rangle=\left\{\left|\downarrow_{2}\right\rangle,\left|\uparrow_{2}\right\rangle\right\}$, that will condition Bob's $U$ rotation to reconstruct the teleported state. In our scheme each operation, $\mathrm{H} \otimes \mathrm{C}-\mathrm{NOT}$ or $\mathrm{C}-\mathrm{NOT} \otimes \mathrm{H}$, is substituted by a single laser pulse.

as it is shown in Fig. 4. Using ionic individual addressing, $\mathrm{C}-\mathrm{NOT}$ gates in the two ion system have been proposed by Cirac and Zoller [9], using 4 laser pulses on a system of ions cooled to the vibrational ground state. In the case of thermal ions, Sørensen and Mølmer [20] have shown that C-NOT gates may be implemented with 7 laser pulses applied on individual ions plus 2 laser pulses applied simultaneously on two ions. The operation equivalent to the product of these two gates is realized physically in a simpler way by means of the interaction in Eq. (7). It realizes simultaneously what the quantum circuit of Fig. 4 shows as a sequence of two operations.

\subsection{Teleportation fidelity}

We study some possible sources of error when considering the teleportation process, say, imprecision in the lasers pulse area and the effects of finite temperature in the initial vibrational state in both traps. We start by considering an ideally well generated Bell state in ions 2 and 3 in trap B, due to the good results of simulations under realistic conditions discussed above. After ion 2 is transported adiabatically from trap $\mathrm{B}$ to trap $\mathrm{A}$, leaving ion 3 alone in trap B, the two traps thermalize in such a way that we may consider a collective two mode thermal state in trap A and a one mode motional thermal state in trap B. When associating a common temperature to both collective modes in trap $\mathrm{A}$, the relation between the CM phonon mean value, $\bar{n}$, and the relative phonon mean value, $\overline{n_{r}}$, is

$$
\bar{n}_{r}=\frac{1}{\left(\frac{1}{\bar{n}}+1\right)^{\frac{\nu_{r}}{\nu}}-1},
$$

where $\nu_{r} / \nu=\sqrt{3}$ in our case. For simplicity, we will assume that trap B thermalizes to the same temperature than trap A. We will apply now the required $\pi / 4-$ pulse on ions 1 and 2, in trap A, with the effective Hamiltonian of Eq. (1). We choose the time of action of the pulse to be $(1+\epsilon) \pi /\left(4\left|\Omega_{0,0}^{1}\right|\right)$, where $\epsilon$ is an imprecision factor in the pulse area and $\Omega_{0,0}^{1}$ is the effective Rabi frequency for the collective vibrational ground state. Clearly, for the considered initial density matrix and for $\epsilon=0$, only the terms associated with the vibrational fundamental mode will evolve, as desired, from the Bell state basis to the completely decoupled basis. The other Fock states in the thermal distribution of trap A will produce different final states, given rise to an unavoidable error source. We measure now the global state of ions 1 and 2 in the disentangled basis. Then, ion 3 is left, up to an eventual local unitary operation depending on the result of the measurement, in the original state of ion 1 . This last operation on ion 3 will introduce the same kind of errors as before, due to the one mode thermal distribution in trap B and the imprecision in the lasers pulse area.

An estimation of teleportation fidelity may be done, as it is shown in Fig. 5, using the relation $F=\operatorname{Tr}\left\{\rho_{3} \rho_{3}^{\prime}\right\}$, where $\rho_{3}$ stands for the ideal teleported state in ion 3 and $\rho_{3}^{\prime}$ for the realistic generated state. In Fig. 5(a), no imprecisions in the lasers pulse area are considered, showing only the effect of the increasing $\bar{n}$ and $\eta$. Fidelity $F$ goes down from unity, under ideal conditions of a pure vibrational ground state and Lamb-Dicke limit, to around 0.985, for larger collective phonon thermal states and higher Lamb-Dicke parameters. In Fig. 5(b), we add a rather large imprecision in the lasers pulse area (5\%) and, nevertheless, obtain a Fidelity $F$ which is never less than 0.975 . We recall that in very recent experiments in the coherent manipulation of two ions [23], typical values of $\bar{n}=0.11$ and $\overline{n_{r}}=0.01$ are reported. We may say, then, that our teleportation protocol is robust under realistic conditions studied here.

\subsection{BBCJPW technicalities}

We would want to discuss now some considerations about the standard teleportation protocol 4 . The principal scope of our scheme is to test reliable teleportation in massive particles. For achieving this goal, some minor technicalities, as if it is easier or not to send the particle instead of teleporting its quantum state, are of no relevance. In fact, if we think in tests of teleportation of photonic polarization states, it would be even easier and quicker to send the photon. In this way, we may argue that the experimental realization of teleportation of internal states in ions may be considered as a fundamental step in the coherent manipulation of quantum information and, for sure, an important task in the implementation of a quantum channel between quantum networks. On the other hand, our proposal considers and respects the importance of the arbitrariness of the state in particle 1 as claimed in the standard teleportation protocol, as long as the teleporting scheme must be universal in the sense of state independent. However, it is of no relevance if a third person (usually called Victor) prepares in private an arbitrary state in particle 1 and gives it to Alice, or if Victor prepares the arbitrary state close to Alice, as she may "close her eyes" politely. What should remain clear, as we have stressed before, is that the implementation of our proposal would lead to reliable teleportation, fact that seems 

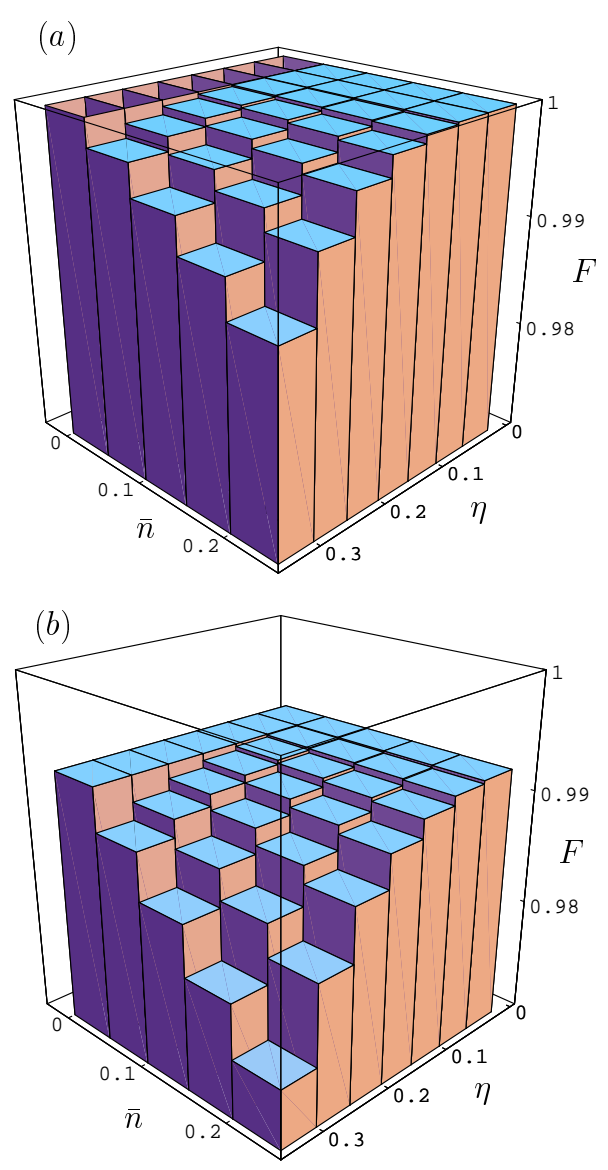

Fig. 5. Fidelity of the teleported state, $F=\operatorname{Tr}\left\{\rho_{3} \rho_{3}^{\prime}\right\}$, as a function of the motional phonon mean number $\bar{n}$ and the CM Lamb-Dicke parameter $\eta$, considering $0 \%$ (a) and $5 \%$ (b) of imprecision in the pulse area, respectively.

impossible for photonic polarization states in the absence of photon-photon interaction.

\section{3 entanglement teleportation and entanglement swapping}

We discuss, briefly and without entering into technical details, natural extensions of our teleportation scheme, i.e., teleportation of an entangled state [4] and the so-called entanglement swapping [24]. For the case of teleportation of an entangled state we need initially two pairs of ions, each pair in a different trap. The first pair in $\operatorname{trap} A$, ions 1 and 2 , is in an arbitrary unknown entangled state, and the second pair, ions 3 and 4, must be in a Bell state. First of all, we transport adiabatically ion 1 to another trap, preserving the entanglement in the internal degrees of freedom. Then, we apply to ions 2, 3 and 4 the same operations used previously for single state teleportation to ions 1, 2 and 3, resulting in the transfer of the initial arbitrary entanglement of ions 1 and 2 to the ions 1 and 4. For achieving entanglement swapping, we consider two traps containing, each one, a pair of ions in a Bell state. For example, we may consider the total initial state $\left|\Phi^{+}\right\rangle_{12}\left|\Phi^{+}\right\rangle_{34}$, that may be rewritten as

$$
\begin{aligned}
& \frac{1}{2}\left\{\left|\Phi^{+}\right\rangle_{14}\left|\Phi^{+}\right\rangle_{23}+\left|\Phi^{-}\right\rangle_{14}\left|\Phi^{-}\right\rangle_{23}\right. \\
&\left.+\left|\Psi^{+}\right\rangle_{14}\left|\Psi^{+}\right\rangle_{23}+\left|\Psi^{-}\right\rangle_{14}\left|\Psi^{-}\right\rangle_{23}\right\}
\end{aligned}
$$

From this equation it is clear that if we project ions 2 and 3 onto any of the four Bell states, we create a similar Bell state between ions 1 and 4 , in spite of the fact that these particles never interacted before. To this end, we will take advantage of the ionic internal state Bell analyzer. For distinguishing the four Bell states in ions 2 and 3 we put this pair into an individual trap, preserving their initial internal correlations with isolated and distant particles 1 and 4 , respectively. Then, we apply on ions 2 and 3 a pulse $\pi / 4$ with the interaction described by the Hamiltonian in Eq. (7), producing the state

$$
\begin{aligned}
& \frac{1}{2}\left\{\left|\Phi^{+}\right\rangle_{14}|\uparrow \uparrow\rangle_{23}+\left|\Phi^{-}\right\rangle_{14}|\downarrow \downarrow\rangle_{23}\right. \\
& \left.+\left|\Psi^{+}\right\rangle_{14}|\uparrow \downarrow\rangle_{23}+\left|\Psi^{-}\right\rangle_{14}|\downarrow \uparrow\rangle_{23}\right\} .
\end{aligned}
$$

Hereafter, we just have to measure the individual internal state of ions 2 and 3 to project particles 1 and 4 onto the corresponding Bell state. Again, we have a theoretical $100 \%$ of success in the process. This was also the case in the previously described teleportation scheme, but different from photonic entanglement swapping proposals 24.

In our view, technical tasks to implement the present teleportation scheme, that will be discussed in the next Section, are similar to those for entanglement teleportation and entanglement swapping. In this sense, we believe that these and other experimental challenges in trapped ions may be envisaged.

\section{Experimental issues}

We now briefly discuss experimental issues, proposing one scenario in which our teleportation scheme could be implemented. While many of the required tasks have been individually demonstrated, others are being actively pursued by experimental groups. For this discussion we borrow heavily on the implementations ideas of Wineland and co-workers 25, as they have been able to successfully address many of the questions raised in this paper. Below is a list and discussion of the required experimental steps. (i) The deterministic generation of an arbitrary one ion internal state $\alpha|\downarrow\rangle+\beta|\uparrow\rangle$ has already been achieved (see for example Ref. [26]). (ii) Ions may be cooled down to such low temperatures that, in a good approximation, we may consider initial density operators of the form $|\downarrow \downarrow\rangle\langle\downarrow \downarrow| \otimes \hat{\rho}_{v i b}$, with $\hat{\rho}_{v i b}$ associated with a distribution were the vibrational ground state is heavily populated 23. (iii) The deterministic generation of a Bell state and the disentangling of the Bell basis, outlined above, may be implemented with state of the art technology through the procedure 
described in detail above. The results of the numerical simulations show that we may be optimistic about the experimental realization of ionic internal Bell states. (iv) Transporting ions from one trap to another requires the construction of novel traps. The envisioned traps would have lithographically deposited electrodes [25] for controlling the displacement of the ions without affecting the internal states. Such traps are being pursued by the group at NIST [27]. These traps should allow for a smooth, almost adiabatic, separation of ions from a single potential well, and the reverse operation, without much heating to the ionic motion. Also, dynamic electric and magnetic transport would not be out of question, so long as the heating is not large and the internal states remain untouched. For that, the switching of electric fields are to be made adiabatic with respect to the internal states, though not necessarily adiabatic with respect to the ions vibrational frequency. Since the theoretical method above for the generation of entanglement is nearly independent of the vibrational quantum number, in the Lamb-Dicke regime, one only has to worry about large heating mechanisms. If such heating exists, it can still be overcome, at added complexity, through the use of sympathetic cooling to cool again the joined ions to low vibrational states, without affecting the relevant ions internal state. For example, sympathetic cooling of ions in a linear Paul trap is in the scope of $\mathrm{W}$. Lange and co-workers [28] to realize quantum computing experiments. In the same row, magnesium ions, suitable for doing quantum logic operations, will be mixed with indium ions, suitable for sympathetic (indirect) cooling of the ions communicated by a collective mode. Recently, J. Hangst and co-workers in Aarhus have performed beautiful experiments demonstrating sympathetic cooling crystallization of trapped ions 32. They were able to keep up to 14 ordered cooled ions by the direct cooling on a single ${ }^{24} \mathrm{Mg}^{+}$ion. (v) The production of the pairs of Raman laser beams and the pulse shaping (power and duration) can be accomplished with the proper use of acousto-optical modulators and RF switches, and the relative phases can be controlled by the path lengths. Laser power levels and timing, while dependent on a chosen experimental system, are estimated to be compatible with what is currently used for Raman excitation of trapped ions (as for example, in Ref.[23]). (vi) Finally, the readout of the internal states of the individual ions 1 and 2 is most easily done by separating them, using the same transport mechanism as above, and using the "electron-shelving" technique 29] for achieving nearly $100 \%$ detection efficiency in each resolved ion. An alternative method, not requiring a separation of the ions, would follow Leibfried's [30] proposal to read out individual electronic states of two or more trapped ions, selected by their rf-micromotion. We also note the work done in R. Blatt's group [31] in Innsbruck, where they were able to directly address individual ions, in an optical transition, by shifting either the ions string or the laser beam with acousto-optical modulators. Unfortunately, their scheme is not suitable for the other tasks required for our proposal as they are in a "weak trap" regime, not being able to perform sideband cooling towards the ground state. More recently, the same group has used a stronger trap and achieved sideband cooling to the ground state on a single $\mathrm{Ca}+$ ion [33]. (vii) Of course, states $|\uparrow\rangle$ and $|\downarrow\rangle$ are either different hyperfine states in the proper magnetic field, such that their energy's difference is field independent, or different metastable electronic states [33,23], making this a robust system with a long coherence time. The specific experimental system will dictate the requirements on laser and trap stabilities, among other experimental parameters.

While the experimental scenario envisioned above is not readily available, we believe it soon will be, as inferred from the latest impressive developments in trapped ion technology.

All arguments presented above convince us that teleportation with massive particles, not speaking about its obvious fundamental interest, are achievable and interesting experimental tasks that will result in several applications in the burgeoning field of quantum information.

\section{5 conclusions}

In summary, we have shown that a reliable teleportation scheme is possible for the internal states of trapped ions and, to this end, we have proposed a suitable Bell analyzer based on an operation that transforms unitarily the Bell basis into a disentangled one. We have presented numerical simulations to show the influence of the technical imprecisions and finite temperature on the desired result. Entanglement teleportation and entanglement swapping are briefly discussed, as they are in fact natural extensions of state teleportation. Finally, a step by step discussion of a probable experimental realization of ionic internal Bell states and teleportation is presented.

This work was partially supported by the Conselho Nacional de Desenvolvimento Científico e Tecnológico (CNPq), Fundação de Amparo à Pesquisa do Estado do Rio de Janeiro (FAPERJ) and the Programa de Apoio a Núcleos de Excelência (PRONEX). We acknowledge useful information from D. Wineland concerning experimental issues, particularly on the implementation of lithographic traps. E. S. would like to thank hospitality and useful discussions, especially about sympathetic cooling and the implementation of our teleportation scheme, to W. Lange and S. Koehler at Max-Planck Institut (Garching, Germany).

\section{References}

1. A. Einstein, B. Podolsky, and N. Rosen, Phys. Rev. A 47, 777 (1935).

2. J. S. Bell, Speakable and Unspeakable in Quantum Mechanics (Cambridge University Press, Cambridge, England, 1987).

3. A. Aspect, P. Grangier, and G. Roger, Phys. Rev. Lett. 49, 1804 (1982).

4. C. H. Bennett, G. Brassard, C. Crépeau, R. Jozsa, A. Peres, and W. Wootters, Phys. Rev. Lett. 70, 1895 (1993). 
5. D. Bouwmeester, J. W. Pan, K. Mattle, M. Eibl, H. Weinfurter, and A. Zeilinger, Nature 390, 575 (1997); D. Boschi, S. Branca, F. De Martini, L. Hardy, and S. Popescu, Phys. Rev. Lett. 80, 1121 (1998).

6. A. Furusawa, J. L. Sørensen, S. L. Braunstein, C. A. Fuchs, H. J. Kimble, and E. S. Polzik, Science 282, 706 (1998).

7. S. Wiesner, Sigact News 15, 78 (1983); A. K. Ekert, Phys. Rev. Lett. 67, 661 (1991); C. H. Benett, G. Brassard, and N. D. Mermin, ibid. 68, 557 (1992); C. H. Benett, ibid. 68, 3121 (1992); A. K. Ekert, J. G. Rarity, P. R. Tapster, and G. M. Palma, ibid. 69, 1293 (1992).

8. D. Deutsch, Proc. R. Soc. London, Ser. A 400, 97 (1985); A. Berthiaume and G. Brassard, in Proceedings of the Seventh Annual IEEE Conference on Structure in Complexity Theory, Boston, 1992 (IEEE, New York, 1992), p. 132.

9. J. I. Cirac and P. Zoller, Phys. Rev. Lett. 74, 4091 (1995). 10. E. Hagley, X. Maître, G. Nogues, C. Wunderlich, M. Brune, J. M. Raimond, and S. Haroche, Phys. Rev. Lett. 79, 1 (1997).

11. B. Englert, M. Löffler, O. Benson, M. Weidinger, B. Varcoe, and H. Walther, Fortschritte der Physik 46, 897 (1998).

12. Q. A. Turchette, C. S. Wood, B. E. King, C. J. Myatt, D. Leibfried, W. M. Itano, C. Monroe, and D. J. Wineland, Phys. Rev. Lett. 81, 3631 (1998).

13. C. A. Sackett, D. Kielpinski, B. E. King, C. Langer, V. Meyer, C. J. Myatt, M. Rowe, Q. A. Turchette, W. M. Itano, D. J. Wineland and C. Monroe, Nature 404, 256 (2000).

14. S. L. Braunstein, A. Mann, and M. Revzen, Phys. Rev. Lett. 68, 3259 (1992).

15. L. Vaidman and N. Yoran, Phys. Rev. A 59, 116 (1999).

16. N. Lütkenhaus, J. Calsamiglia, and K.-A. Suominen, Phys. Rev. A 59, 3295 (1999).

17. L. Davidovich, N. Zagury, M. Brune, J. M. Raimond, and S. Haroche, Phys. Rev. A 50, R895 (1994).

18. J. I. Cirac and A. S. Parkins, Phys. Rev A 50, R4441 (1994).

19. E. Solano, R. L. de Matos Filho, and N. Zagury, Phys. Rev. A 59, R2539 (1999); 61, 029903(E) (2000).

20. A. Sørensen and K. Mølmer, Phys. Rev. Lett. 82, 1971 (1999). K. Mølmer and A. Sørensen, Phys. Rev. Lett. 82, 1835 (1999).

21. Anders Sørensen and Klaus Mølmer, Phys. Rev. A 62, 022311 (2000).

22. J. Preskill, Quantum Information and Quantum Computation (http://www.theory.caltech.edu/ preskill/ph229).

23. B. E. King, C. S. Wood, C. J. Myatt, Q. A. Turchette, D. Leibfried, W. M. Itano, C. Monroe, and D. J. Wineland, Phys. Rev. Lett. 81, 1525 (1998).

24. Jian-Wei Pan, Dik Bowmeester, Harald Weinfurter and Anton Zeilinger, Phys. Rev. Lett. 80, 3891 (1998).

25. D. J. Wineland, C. R. Monroe, W. M. Itano, D. Leibfried, B. E. King, and D. M. Meekhof, NIST J. Research 103 (3), 259 (1998) (Available at http://nvl.nist.gov/pub/nistpubs/jres/jres.htm); D. J. Wineland, C. Monroe, W. M. Itano, D. Kielpinski, B. E. King, C. J. Myatt, Q. A. Turchette, and C. S. Wood, in Proceedings of the 6th International Symposium on Foundations of Quantum Mechanics in the Light of New Technology, edited by K. Fujikawa, and Y. A. Ono (Elsevier Science, Amsterdam, in press) (Available at quant-ph/9809028).

26. D. M. Meekhof, C. Monroe, B. E. King, W. M. Itano, and D. J. Wineland, Phys. Rev. Lett. 76, 1796 (1996).
27. D. Wineland at NIST (Boulder, USA), private communication.

28. W. Lange at Max-Planck Institut (Garching, Germany), private communication.

29. W. Nagourney, J. Sandberg, and H. Dehmelt, Phys. Rev. Lett. 56, 2797 (1986).

30. D. Leibfried, Phys. Rev. A 60, R3335 (1999).

31. H.C. Nägerl, D. Leibfried, H. Rohde, G. Thalhammer, J. Eschner, F. Schmidt-Kaler, and R. Blatt, Phys. Rev. A 60, 145 (1999).

32. P. Bowe, L. Hornekaer, C. Brodersen, M. Drewsen, J.S. Hangst, and J.P. Schiffer, Pys. Rev. Lett. 82, 2071 (1999).

33. Ch. Roos, Th. Zeiger, H. Rohde, H.C. Nägerl, J. Eschner, D. Leibfried, F. Schmidt-Kaler, and R. Blatt, Phys. Rev. Lett. 83, 4713 (1999). 